\title{
Lithium Associated Hyperparathyroidism: An Evidence Based Surgical Approach
}

\author{
Umashankar K. Ballehaninna ${ }^{1,2}$, Steven M. Nguyen ${ }^{3}$, Ronald S. Chamberlain ${ }^{1,3,4}$ \\ ${ }^{1}$ Department of Surgery, Saint Barnabas Medical Center, Livingston, USA \\ ${ }^{2}$ Department of Surgery, Maimonides Medical Center, Brooklyn, USA \\ ${ }^{3}$ Department of Surgery, University of Medicine and Dentistry of New Jersey, Newark, USA \\ ${ }^{4}$ Saint George's University, School of Medicine, West Indies, Grenada \\ E-mail: rchamberlain@sbhcs.com
}

Received May 17, 2011; revised August 15, 2011; accepted October 17, 2011

\begin{abstract}
Background: Long-term lithium use in psychiatric patients may lead to lithium associated hyperparathyroidism (LAH). Although anecdotal case reports have appeared, an evidence based algorithm for management of LAH is lacking. Methods: A comprehensive literature search was performed (1973-2010) using PubMed with keywords; "lithium" "hypercalcemia" "hyperparathyroidism" "sestamibi" "intra-operative parathyroid hormone (IOPTH) monitoring" "parathyroidectomy" and "medical management". All English language publications addressing etiology and clinical management issues concerning LAH were critically analyzed. Results: Lithium associated hyperparathyroidism occurs in $4.3 \%-6.3 \%$ of chronic lithium users compared to the general population which has an incidence of $0.5 \%-1 \% .194$ cases of LAH have been reported which includes 10 patients $(5 \%)$ treated medically and 170 patients $(88 \%)$ who underwent parathyroidectomy. No details were available for 14 patients (7\%). Among parathyroidectomy patients, 104 (59\%) had adenomatous disease and $66(39 \%)$ had multiglandular hyperplasia. Preoperative localization studies were utilized in only 22 patients (13\%) and IOPTH monitoring was reported in only 3 studies (32 patients, 19\%). Among surgical patients, bilateral neck exploration (BNE) was the most common approach performed in 162 patients (95\%); focused neck exploration was utilized in only 8 patients $(5 \%)$. Parathyroidectomy normalized LAH biochemical changes in nearly all patients $(90 \%-97 \%)$ in the early post-operative period, but recurrent hyperparathyroidism occurred in $8 \%-42 \%$ of patients. Conclusion: LAH is an under appreciated and poorly understood endocrine disorder. LAH has a higher incidence of multiglandular disease and bilateral neck exploration is mandatory in majority for disease control. Nonsurgical approaches may be useful in select patients on short-term lithium therapy.
\end{abstract}

Keywords: Lithium, Hypercalcemia, Hyperparathyroidism, Sestamibi Intra-Operative Parathyroid Hormone (IOPTH) Monitoring, Parathyroidectomy, Medical Management

\section{Introduction}

Lithium Carbonate $\left(\mathrm{Li}^{+}\right)$is the preferred and most efficacious therapy for acute treatment and maintenance therapy for bipolar depressive disorder, and is a useful adjunct in unipolar depression [1,2]. Long-term lithium therapy is associated with multiple endocrine and metabolic alterations such as hyperthyroidism, hypothyroidism, reduced bone mineral density (BMD), osteopenia, as well as several gastrointestinal (nausea, constipation), cardiovascular (bradyarrythmias), renal (nephrocalcinosis, re- duced glomerular filtration rate (GFR), polyuria, nephrogenic diabetes insipidus) and psychosomatic adverse effects (weakness, fatigue, depression). Chronic lithium therapy is also associated with hyperparathyroidism (LAH) characterized by hypercalcemia, hypermagnesemia, reduced urinary calcium, and elevated serum PTH levels often with enlarged parathyroid gland(s) [3,4]. Rare case reports and a limited number of reviews have yielded inadequate and conflicting data concerning the ideal treatment of LAH [4-6]. This article provides a systematic review of LAH, addresses current controversies, and pro- 
poses an algorithmic approach to LAH treatment.

\subsection{Methods/Materials}

All English language LAH publications for the years 1973 to 2010 which addressed the epidemiology, biochemical association, etiology, pathogenesis, clinical presentation, preoperative imaging studies, intraoperative parathyroid hormone monitoring (IOPTH), surgical management (parathyroidectomy) and surveillance were collected and analyzed. The literature search was performed utilizing PubMed with the following keywords; "lithium" "hypercalcemia" "hyperparathyroidism" "sestamibi" "IOPTH" "parathyroidectomy" "medical management"and "cinacalcet".

\subsection{Results}

Lithium use is associated with an increased incidence of hyperparathyroidism $(4.3 \%-6.3 \%)$ and a female prepon- derance (F: M 4:1) compared to primary hyperparathyroidism in the general population $(0.5 \%-1 \%$ and $\mathrm{F}$ : $\mathrm{M}$ $3: 1$ ). A total of 194 cases of LAH have been reported in English language literature of which 170 patients $(88 \%)$ were treated by surgical resection (parathyroidectomy), 10 patients $(5 \%)$ were managed medically and no details are available for 14 patients (7\%). (Tables 1 and 2) Among those who had parathyroidectomy, 104 patients (61\%) had adenomatous disease and 66 patients (39\%) had multigland parathyroid hyperplasia compared to primary hyperparathyroidism (PHPT) in the general population in whom single adenoma is seen in $85 \%$ and multigland hyperplasia is identified in $15 \%$. Among $170 \mathrm{LAH}$ patients undergoing parathyroidectomy, preoperative localization studies (sestamibi scan, neck ultrasound, CT scan) were utilized in only 22 patients (14\%) and IOPTH monitoring was reported in only 3 studies (32 patients, 19\%).

Bilateral neck exploration was the most common surgical approach utilized and was utilized in 162 patients (95\%), whereas focused neck exploration was performed

Table 1. Published studies on the surgical management of lithium associated hyperparathyroidism (1973-2010).

\begin{tabular}{|c|c|c|c|c|c|c|}
\hline \multirow[b]{2}{*}{ Author, Year } & \multirow[b]{2}{*}{ Total $(\mathrm{N}=)$} & \multirow{2}{*}{$\begin{array}{l}\text { Surgically Treated } \\
(\mathrm{N}=)(\%)\end{array}$} & \multicolumn{2}{|c|}{ Adenoma } & \multirow[b]{2}{*}{$\begin{array}{l}\text { Four gland hyperplasia } \\
\qquad(\mathrm{N}=)(\%)\end{array}$} & \multirow[b]{2}{*}{$\begin{array}{l}\text { Remarks (Surgical approach, } \\
\text { BNE, IOPTH monitoring) }\end{array}$} \\
\hline & & & $\begin{array}{c}\text { Single } \\
(\mathrm{N}=)(\%)\end{array}$ & $\begin{array}{l}\text { Double } \\
(\mathrm{N}=)(\%)\end{array}$ & & \\
\hline Garfinkel et al. [10], 1973 & 1 & $1(100 \%)$ & $1(100 \%)$ & - & - & First reported case, BNE \\
\hline Ananth et al. [20], 1983 & 3 & $2(67 \%)$ & $2(67 \%)$ & - & - & BNE, 1 patient-details-NA \\
\hline Stancer et al. [15], 1989 & 8 & $3(37.5 \%)$ & $1(12.5 \%)$ & - & $2(25 \%)$ & BNE, 5 patients-details-NA \\
\hline Nordenstrom et al. [19], 1992 & 6 & $6(100 \%)$ & $1(17 \%)$ & - & $5(83 \%)$ & BNE \\
\hline Bendz et al. [8], 1996 & 8 & $8(100 \%)$ & $3(37.5 \%)$ & $2(25 \%)$ & $3(37.5 \%)$ & Point prevalence of LAH-3.6\% \\
\hline McHenry et al. [3], 1996 & 25 & $17(68 \%)$ & $12(48 \%)$ & - & $5(20 \%)$ & BNE, 8 patients-details-NA \\
\hline Wolf et al. [21], 1997 & 1 & $1(100 \%)$ & - & - & $1(100 \%)$ & BNE \\
\hline deCelis et al. [22]. 1998 & 1 & $1(100 \%)$ & $1(100 \%)$ & - & - & BNE \\
\hline Abdullah et al. [17], 1999 & 11 & $11(100 \%)$ & $6(55 \%)$ & $3(27 \%)$ & $2(18 \%)$ & $\mathrm{BNE}, 1$ recurrence \\
\hline Awad et al. [5], 1999 & 15 & $15(100 \%)$ & $11(73 \%)$ & $3(20 \%)$ & $1(7 \%)$ & BNE \\
\hline Hundley et al. [18], 2005 & 12 & $12(100 \%)$ & $6(50 \%)$ & $3(25 \%)$ & $3(25 \%)$ & IOPTH monitoring \\
\hline Carchman et al. [16], 2008 & 16 & $16(100 \%)$ & $12(75 \%)$ & - & $4(25 \%)$ & $\begin{array}{l}\text { IOPTH monitoring, } 8 \text { patients } \\
\text { had focused neck exploration }\end{array}$ \\
\hline Rizwan et al. [27], 2009 & 1 & $1(100 \%)$ & - & - & $1(100 \%)$ & BNE \\
\hline Szalat et al. [12], 2008 & 4 & $4(100 \%)$ & $1(25 \%)$ & $2(50 \%)$ & $1(25 \%)$ & $75 \% \mathrm{MGH}$, IOPTH monitoring \\
\hline Jarhult et al. [14], 2010 & 71 & $71(100 \%)$ & $32(45 \%)$ & $2(3 \%)$ & $37(52 \%)$ & $\mathrm{BNE}, 42 \%$ had recurrence \\
\hline Current study, 2010 & 1 & $1(100 \%)$ & - & - & $1(100 \%)$ & IOPTH, BNE \\
\hline Overall & $184^{*,+}$ & $170(92 \%)$ & $89(52 \%)$ & $15(7 \%)$ & $66(39 \%)$ & $\begin{array}{c}\text { Multigland (adenomas and } \\
\text { hyperplasia seen in } 46 \%),{ }^{*} \text { No } \\
\text { details available for } 14(7 \%) \\
\text { patients, }{ }^{+} 10 \text { patients managed } \\
\text { medically see table } 2 .\end{array}$ \\
\hline
\end{tabular}

Abbreviations: N, number of patients; BNE, bilateral neck exploration; MGH, Multiglandular hyperplasia; IOPTH, intraoperative parathyroid hormone monitoring; LAH, lithium associated hyperparathyroidism; NA, not available. Note: Published studies on the surgical management of LAH document that a single adenoma is the most common LAH associated pathology (61\%). However, increased prevalence of double adenomas (7\%) and multigland hyperplasia (39\%) suggests that bilateral neck exploration is necessary in the majority of LAH patients $(96 \%)$. Pre-operative localization studies and IOPTH monitoring may have utility in LAH patients, however data on their value is limited $(\mathrm{N}=22,33$ respectively). 
Table 2. Published studies on the medical management of lithium associated hyperparathyroidism (1973-2010).

\begin{tabular}{|c|c|c|}
\hline Author, Year & $\mathrm{N}=$ & Management \\
\hline Gama et al. [3], 1999 & 1 & Resolution of LAH after lithium was discontinued \\
\hline Sloand et al. [23], 2006 & 2 & Modest response while lithium was continued in one patient \\
\hline Khandawala et al. [24], 2006 & 1 & Resolution of LAH after lithium was discontinued \\
\hline Rifai et al. [25], 2006 & 1 & Discontinuation of $\mathrm{Li}^{+}$, normocalcemia achieved by cinacalcet \\
\hline Duggal et al. [26], 2007 & 1 & Resolution of LAH after lithium was discontinued \\
\hline Gregoor et al. [34], 2007 & 3 & Cinacalcet therapy ameliorated LAH \\
\hline Szalat et al. [12], 2009 & 1 & Postoperative recurrence - hypercalcemia corrected with cinacalcet, however elevated PTH persisted. \\
\hline
\end{tabular}

Abbreviations: $\mathrm{LAH}$, lithium associated hyperparathyroidism, $\mathrm{Li}^{+}$, Lithium, PTH, parathyroid hormone. Note: Medical management (withdrawal of lithium and/or use of cinacalcet) has been successfully used to treat a small number of LAH patients.

in $8(5 \%)$ patients. The latter approach was utilized significantly less often in LAH when compared to PHPT patients in whom the published unilateral focused neck exploration rate is $80 \%-95 \%$ [7]. This change likely reflects the increased incidence of four-gland hyperplasia in LAH. Parathyroidectomy normalized LAH associated biochemical changes in nearly all patients $(67 \%-100 \%)$ in the early post-operative period, however at a median long-term follow-up of 6.3 years $8 \%$ - $42 \%$ patients experienced recurrence.

\section{Case Review}

A 64 year-old female with a past medical history of type II diabetes mellitus, hypertension, hypercholesterolemia, and bipolar depression was referred for surgical evaluation due to persistent hypercalcemia. She had been treated with lithium (300 mg BID) for four years. Over the prior three years, the patient had mild elevations of her serum calcium level (normal range; 10 - $11 \mathrm{mg} / \mathrm{dL}$ ) which rose to $11.7 \mathrm{mg} / \mathrm{dL}$ and $12.3 \mathrm{mg} / \mathrm{dL}$ over a two months duration. Symptomatically she complained of decreased memory and mental clarity. Her diabetes was well controlled (hemoglobin A1C level of 6\%) with no evidence of retinopathy or nephropathy. A sestamibi parathyroid scan localized a parathyroid adenoma at the mid pole of the left lobe of the thyroid gland. The patient was scheduled for a PTH directed left parathyroidectomy under local anesthesia. Pre-operative PTH level was $147 \mathrm{pg} / \mathrm{ml}$ (normal range; $10-55 \mathrm{pg} / \mathrm{ml}$ ). At exploration a large cystic structure was identified on the anterior surface of the left lower thyroid lobe which obscured the surgical field. The procedure was converted to general anesthesia and exploration was expanded to the upper pole of the thyroid where an enlarged left superior parathyroid gland was identified and removed. On pathologic review the gland was hypercellular and weighed $286 \mathrm{mg}$. A postexcision intact PTH level measured 15 minutes after excision remained high $(89 \mathrm{pg} / \mathrm{ml})$, and a four-gland ex- ploration of the neck was performed. Enlarged right superior $(866 \mathrm{mg})$ and inferior parathyroid glands $(256 \mathrm{mg})$ were identified and removed. The left inferior parathyroid was partially resected and intraoperative pathologic evaluation identified this gland as also hypercellular. The remaining portion of the left inferior parathyroid was autotransplanted into the left sternocleidomastoid muscle. The post-operative course was uncomplicated and the patient was discharged home on day 2 with a serum calcium level of $9.1 \mathrm{mg} / \mathrm{dl}$ and a PTH level of $15.8 \mathrm{pg} / \mathrm{mL}$.

\section{Discussion}

A causal association between chronic lithium therapy and hyperparathyroidism has been conclusively demonstrated. As noted earlier, patients on long-term lithium therapy have a 4 - 6 fold increased incidence of hyperparathyroidism compared to the general population [5,7]. Bendz et al. identified a $2.7 \%$ increased point prevalence of hyperparathyroidism among 124 patients on long-term lithium therapy ( $>15$ years), while Mallette et al. reviewed studies involving 309 patients on chronic lithium therapy and identified 37 patients (12\%) with hypercalcemia and 18 patients (16\%) with elevated PTH levels [8-10]. McHenry et al. have reported that $80 \%$ of patients treated with lithium for 6 to 24 months experience a $10 \%$ increase in serum calcium levels. Christiansen et al. re- ported that both serum calcium and PTH levels increased by $30 \%$ in the same population, and several other authors have also reported a $10 \%-60 \%$ increased prevalence of hypercalcemia and hyperparathyroidism in patients taking lithium [11-14].

LAH is associated with characteristic biochemical changes consisting of an elevated serum levels of calcium, magnesium and parathyroid hormone and decreased urinary calcium and cyclic adenosine monophosphate (CAMP) levels. These changes are similar to familial hypocalciuric hypercalcemia $(\mathrm{FHH})$, but distinct from PHPT in which urinary calcium excretion is increased 
[15]. A large number of mechanisms have been proposed to account for the biochemical changes seen in LAH. (Table 3) To briefly summarize, lithium competitively antagonizes calcium sensing receptors (CaSR) and raises the threshold of serum calcium necessary to inhibit PTH secretion. This action not only increases parathyroid hormone secretion, but also exerts a multitude of systemic effects on the parathyroid glands, renal tubules and bone metabolism resulting in parathyroid gland hyperplasia and biochemical changes. Lithium is also known to accentuate adenoma formation by increasing PTH gene transcription, which may unmask hyperparathyroidism in patients with sub-clinical pre-existing adenomas once lithium is initiated.

As a consequence of the aforementioned systemic effects, it seems reasonable to conclude that lithium use should be associated with multiglandular parathyroid hyperplasia. However in most series, a solitary parathyroid adenoma was the most common LAH associated pathology. Awad et al. reported parathyroid adenomas in 14 out of 15 patients (93\%) with LAH, and Carchman et al. identified adenomas in 12 out of $16 \mathrm{LAH}$ patients $(75 \%)$ $[5,16]$. Similarly, in a series of 12 LAH patients, Hundley et al. noted parathyroid adenomas in 9 patients (75\%), while Bendz et al. reported adenoma in $72.5 \%$ of LAH patients (5 out of 8$)[8,18]$. Of note however, many authors have also described a higher incidence of multiglandular hyperplasia. Nordenstrom et al. identified multiglandular hyperplasia (MGH) in $83 \%$ of LAH patients (5 out of 6) [19]. Likewise, Jarhult et al. studied 71 patients with LAH and reported MGH in 37 patients (52\%) [14]. Table 1 summarizes all reported LAH cases and demonstrates that parathyroid adenoma was the most common pathology identified in 104 out of 170 patients
(61\%) following parathyroidectomy [3,5,8,23-26]. (Table 1) In comparison, PHPT is associated with a single adenoma $85 \%$, multigland hyperplasia (MGH) $15 \%$ and parathyroid carcinoma in $1 \%$ of patients [7]. It is important to point out that there is also an increased incidence rate of multigland hyperplasia (MGH) in LAH (39\%, 66 of 170 patients) compared to the general population with PHPT (15\%). This increased MGH incidence is likely attributable to the systemic action of lithium, and as a result may effect interpretation of localization studies (increased false negative rate, poor sensitivity) and surgical planning (increased need of bilateral neck exploration) $[3,4,6,18]$.

The initial management of LAH is medical intervenetion which includes discontinuation of lithium or alternative treatment such as atypical antipsychotics and calcimimmetics. (Table 2) Data relating to the efficacy of LAH medical management is available for only 10 of 194 reported LAH patients (5\%). As such it is difficult to make any meaningful conclusions [12,23-26,34]. In many LAH patients, discontinuation of lithium carbonate is not medically feasible due to increased propensity of relapse [35,36]. Although serum $\mathrm{Ca}^{2+}$ and PTH levels tend to normalize within 4 - 6 weeks after lithium discontinuation, this change is mostly seen in patients receiving lithium for a relatively short-term defined as 5 10 years, or in those with only mild hypercalcemia [4]. Khandawala et al. reported on one patient who was taking lithium for 5 years, discontinuation of $\mathrm{Li}^{+}$resulted in normocalcemia and a normal PTH level which was maintained at 5 months follow-up [24]. Dugal et al. reported on a single patient who had been on lithium therapy for 10 years, in whom discontinuation of lithium and simultaneous saline diuresis with furosemide also achi-

Table 3. Proposed mechanisms of action responsible for Lithium associated hyperparathyroidism (LAH).

\begin{tabular}{|c|c|}
\hline Mechanism of action & Explanatation \\
\hline Antagonism of cations $[28,29]$ & $\begin{array}{l}\mathrm{Li}^{+} \text {competitively antagonizes cations at calcium receptor at several locations - kidney, bone and } \\
\text { parathyroid gland-causing acute hypercalcemia and hyper-magnesemia }\end{array}$ \\
\hline Mitogenic action $[16,32,33]$ & $\begin{array}{l}\mathrm{Li}^{+} \text {exacerbates a pre-existing adenoma or PHPT - this occurs especially in patients presenting with } \\
\text { psychiatric symptoms - the lithium therapy worsens hypercalcemia by promoting PTH overproduc- } \\
\text { tion. Nearly } 55 \% \text { of LAH have parathyroid adenomas (single or double). }\end{array}$ \\
\hline $\begin{array}{l}\uparrow \text { Parathyroid volume and parathyroid } \\
\text { hyperplasia }[2,5,11]\end{array}$ & $\begin{array}{l}\text { LAH is associated with multiglandular hyperplasia in } 25 \%-55 \% \text { cases (Table } 1 \text { ) compared to } 15 \% \\
\text { in PHPT, which is attributed to direct stimulation of parathyroid gland by lithium. In patients on } \\
\text { chronic lithium therapy neck ultrasound demonstrates increased parathyroid gland volume. } \\
\text { Mechanism: } \\
\text { 1) Inhibits inosital monophosphate (IMP) which regulates intracellular calcium exposure to nuclear } \\
\text { CASR in PTH cells, thus altering the set point to turn-off PTH gene transcription. } \\
\text { 2) } \mathrm{Li}^{+} \text {inhibits glycogen synthase kinase-3b (GSK-3b) responsible for inhibition of PTH gene tran- } \\
\text { scription, thereby leading to overproduction of PTH. }\end{array}$ \\
\hline
\end{tabular}

Abbreviations: PHPT-primary hyperparathyroidism; PTH, parathyroid hormone; $\mathrm{Li}^{+}$, lithium; CASR, calcium sensing receptor; IMP, inosital monophosphate; GSK-3b, glycogen synthase kinase-3b. Note: Several different mechanisms have been proposed by which lithium may cause hypercalcemia, $\uparrow$ PTH levels, $\uparrow$ magnesium and $\downarrow$ urinary calcium levels. 
eved normocalcemia [26]. In contrast, drug withdrawal from patients on chronic lithium therapy ( $>10$ years), has a lower rate of hypercalcemia resolution [4]. Carchman et al. noted that even after discontinuation of lithium for more than 5 months, 8 out of 16 patients on chronic lithium therapy experienced persistent hypercalcemia [16]. In LAH patients who have mild hypercalcemia or were asymptomatic, alternative medical therapy such as valproate, carabamazepine or atypical antipsychotics (riseperidone) may be treatment alternatives, however at present there is very limited data to assess their efficacy in LAH patients $[2,4]$.

Recently, a new class of drugs (calcimimmetics, example: cinacalcet) has been successfully used to treat LAH with or without discontinuation of lithium [34]. (Table 3) Calcimimmetics activate calcium-sensing receptors (blocked by lithium) which in turn reduce PTH secretion and prevent parathyroid hyperplasia. To date, a biochemical remission was achieved in 6 cases using cinacalcet among patients on chronic lithium therapy [12, $23,25,34]$. Gregoor et al. reported resolution of LAH in 3 patients using cinacalcet while lithium was continued [34]. Szalat et al. used cinacalcet in an LAH patient who had a post-operative recurrence of hyperparathyroidism and reported resolution of hypercalcemia despite persistently elevated PTH levels [12]. Although above results are intriguing, the consistently reproducible results of parathyroidectomy have made surgical therapy the mainstay of LAH management, particularly among symptomatic patients with moderate to severe hypercalcemia, and those in whom discontinuation of $\mathrm{Li}^{+}$is not feasible or has failed [4].

The most common indication for parathyroidectomy in LAH patients include worsening psychosomatic $(50 \%$ $60 \%$ ) (fatigue, weakness, decreased concentration, shortterm memory loss, depression), bone (30\% - 40\%) (bone pain, reduce bone mineral density, osteopenia or osteoporosis), gastrointestinal symptoms $(20 \%-30 \%)$ (constipation, nausea or pancreatitis), cardiac dysrhythmias and renal symptoms ( $1 \%$ - 5\%) (decreased GFR, decreased creatinine clearance or nephrocalcinosis) $[3,5,12,15,18]$.

Today, preoperative localization studies (ultrasound of neck, sestamibi scan and computerized tomography (CT) scan of neck) have been routinely incorporated into the management algorithm of PHPT patients, and resulted in the near universal use of focused neck exploration $(90 \%$ - 95\%) for this group. However, there is a paucity of data supporting the utility of preoperative localization studies in LAH patients. Among 195 LAH patients who underwent parathyroidectomy, preoperative localization studies were utilized in only 22 patients $(13 \%)$. In the largest series involving 18 patients, Carchman et al. used sestamibi scan or ultrasound in 16 patients (94\%). Imaging studies predicted a single adenoma in 10 patients, $2(20 \%)$ of which had MGH at exploration [19]. In the same study, pre-operative localization studies identified $\mathrm{MGH}$ in 4 patients, however 2 of these patients subsequently were found to have only a single adenoma at exploration. Limited or focused neck exploration was possible in only 8 of 16 patients $(50 \%)$ aided by both pre-operative localization studies and IOPTH monitoring. Szalat et al. have also reported using preoperative neck ultrasound and parathyroid scan which identified solitary uptake in 3 of 4 cases. However, at exploration IOPTH testing failed to return to baseline or decrease by $>50 \%$ (positive response), and all patients required bilateral neck exploration (BNE) [12]. Despite the small number of patients included in these studies, they nevertheless highlight the poor sensitivity and limited utility of pre-operative localization studies for precise localization of pathogically enlarged parathyroid glands in LAH and in planning surgical approach [6].

Parathyroidectomy is the main stay of treatment for LAH patients. Given the reported higher incidence of multiglandular hyperplasia (39\%) and double adenomas $(7 \%)$, it is not surprising that bilateral neck exploration (BNE) is the most commonly reported surgical approach in LAH. Among 170 patients with LAH, BNE was used in 162 patients $(95 \%)$, whereas focused neck exploration was performed in only 8 patients $(5 \%)$. In the largest reported LAH series, Jarhult et al. utilized BNE in all but 2 of 71 patients who underwent parathyroidectomy [14].

As noted earlier, intraoperative parathyroid hormone monitoring (IOPTH) has been extensively validated in patients with PHPT, and prompted more limited surgical exploration [7]. To date, only 3 studies involving LAH patients $(\mathrm{N}=32)$ have assessed the usefulness of IOPTH monitoring $[12,18,19]$. Hundley et al. utilized IOPTH monitoring in 12 patients with LAH, of which 6 patients underwent MGH resection prompted by IOPTH monitoring [18]. In a study of 16 patients, Carchman et al. performed focused parathyroidectomy in 8 patients, while 8 patients underwent BNE guided by IOPTH monitoring [19]. Of note, these authors were able to perform a limited surgical exploration in $50 \%$ of LAH patients, compared to a $5 \%$ rate in other LAH series $[12,14]$.

Parathyroidectomy results in biochemical remission leading to normocalcemia and normal PTH levels in the immediate post-operative period and improves psychosomatic symptoms in $90 \%$ - 97\% of LAH patients. Awad et al. observed eucalcemia in 15 out of 16 patients who had parathyroidectomy for LAH; however, one patient subsequently developed recurrence at 2 years and underwent neck re-exploration with removal of an additional parathyroid adenoma [5]. At a median follow-up of 6 months (5 - 50 months range), Carchman et al. noted that 
all patients were eucalcemic, however 2 patients had mild PTH elevation [19]. In contrast, Jarhult et al. reported that among $71 \mathrm{LAH}$ patients who underwent parathyroidectomy, 30 patients $(42 \%)$ had persistent or recurrent hyperparathyroidism at a median follow-up of 6.3 years [14]. In this series, six out of 30 patients with persistent/recurrent HPT underwent surgical re-exploration but only 2 patients were cured of hypercalcemia, one had permanent hypocalcaemia.

\section{An Evidence Based Algorithm for the Management of LAH}

Knowledge of the higher prevalence of hyperparathyroidism in chronic lithium users coupled with published data on the medical and surgical treatment of LAH provides a basis for developing an algorithmic approach for the treatment of LAH. (Figure 1) Serum calcium levels should be drawn before initiating lithium therapy (to rule-

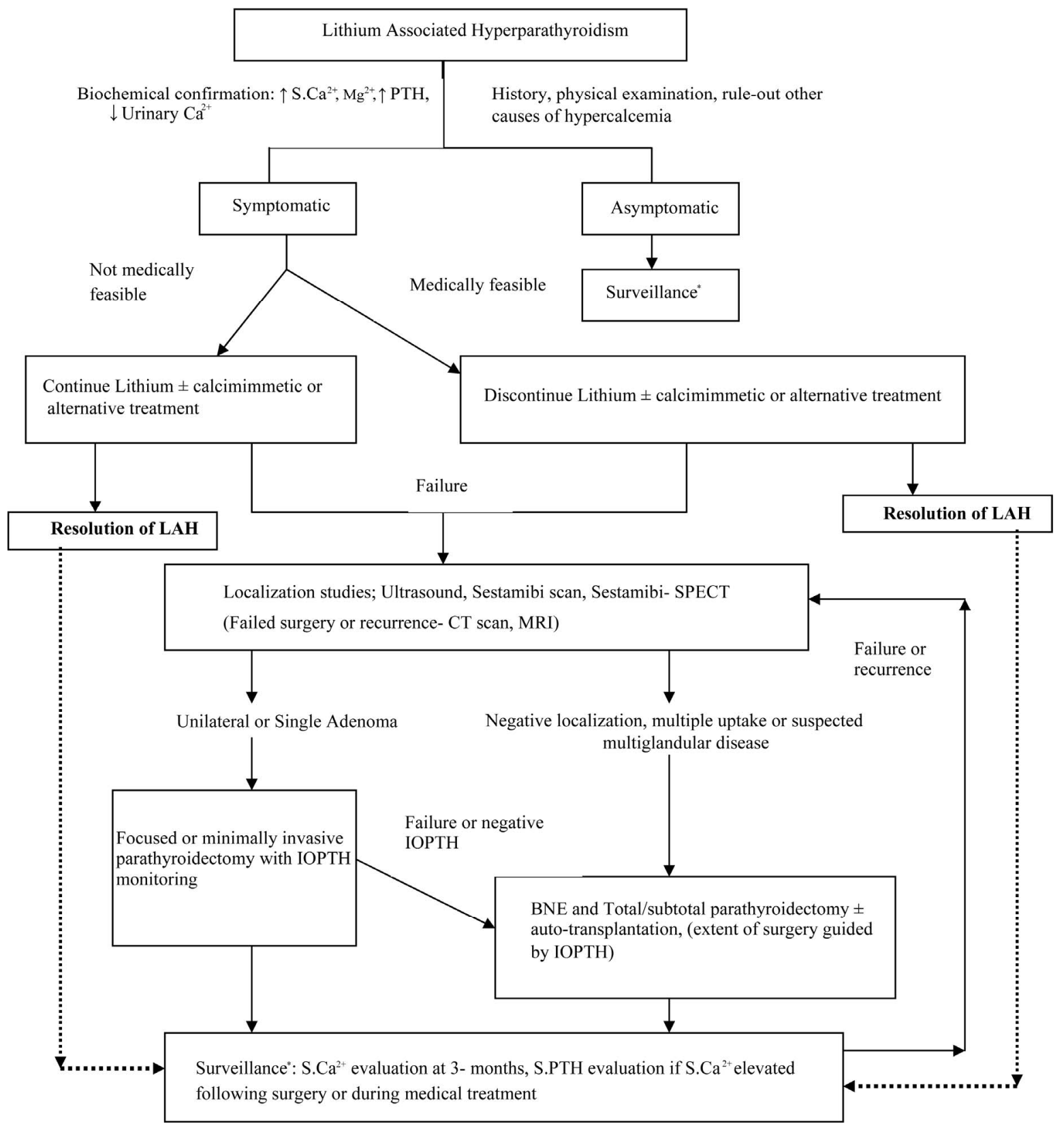

Figure 1. An evidence based algorithm for the management of lithium associated hyperparathyroidism. Abbreviations: S. $\mathrm{Ca}^{2+}$, Serum calcium; S. $\mathrm{Mg}^{2+}$, Serum magnesium; S. PTH-serum parathormone; CVS-cardiovascular system; GI-gastrointestinal system; 4D-CT, 4-dimensional computed tomography; SPECT), Single Photon Emission Computed Tomography scan; IOPTH, Intra-operative parathormone monitoring; BNE, bilateral neck exploration. 
out pre-existent primary HPT) and monitored within 4 6 weeks of beginning lithium therapy, and every 3 months thereafter. Serum PTH levels should be measured whenever hypercalcemia is identified. A confirmatory diagnosis of LAH necessitates ruling-out other causes of HPT. Asymptomatic patients with mild to moderate hypercalcemia may be monitored at regular intervals. The National Institute of Health (NIH) guidelines for the treatment of PHPT are also useful in LAH patients in regards to patient selection for parathyroidectomy [37]. (Figure 1) When medically feasible lithium should be discontinued or alternative therapy substituted (valproate, carbamazepine or atypical antipsychotics) in selected LAH patients. Cinacalcet hydrochloride, a calcimimmetic agent, may have modest efficacy in treating LAH, and be beneficial to patients in whom discontinuation of lithium is not medically feasible or when surgery is contraindicated. In the vast majority of symptomatic patients, and in those in whom either discontinuation of lithium is not possible or when alternative therapy has failed to correct hypercalcemia, parathyroidectomy is required. While preoperative localization studies may provide useful information regarding the number and location of enlarged parathyroid glands, the increased incidence of multiglandular disease in LAH, as well as a higher risk of recurrent or persistent HPT, mandates meticulous surgical exploration to visualize and remove all pathologically enlarged gland(s). Whereas a single parathyroid adenoma is found in the majority $(61 \%)$ of LAH patients, limited or focused surgical exploration may be attempted when preoperative localization studies suggest feasibility, but must be further confirmed with IOPTH monitoring. A very high incidence of persistent and recurrent disease in LAH patients necessitates long-term follow-up and strict surveillance (Figure 1).

In summary, lithium use in psychiatric patients may be associated with hypercalcemia and elevated PTH levels referred to as lithium associated hyperparathyroidism (LAH). An algorithmic approach and strict post-operative surveillance protocols may improve treatment success and identify recurrent or persistent disease (Figure 1).

\section{References}

[1] A. M. Nivoli, A. Murru and E. Vieta, "Lithium: Still a Cornerstone in the Long-Term Treatment in Bipolar Disorder?" Neuropsychobiology, Vol. 62, No. 1, 2010, pp. 27-35. doi:10.1159/000314307

[2] W. Coryell, "Maintenance Treatment in Bipolar Disorder: A Reassessment of Lithium as the First Choice," Bipolar Disorders, Vol. 11, No. 2, 2009, pp. 77-83. doi:10.1111/j.1399-5618.2009.00712.x

[3] C. McHenry and K. Lee, "Lithium Therapy and Disorders of the Parathyroid Glands," Endocrine Practice, Vol. 2, No. 2, 1996, pp. 103-109.

[4] B. D. Saunders, E. F. Saunders and P. G. Gauger, "Lithium Therapy and Hyperparathyroidism: An EvidenceBased Assessment," World Journal of Surgery, Vol. 33, No. 11, 2009, pp. 2314-2323. doi:10.1007/s00268-009-9942-4

[5] S. S. Awad, J. Miskulin and N. Thompson, "Parathyroid Adenomas versus Four-Gland Hyperplasia as the Cause of Primary Hyperparathyroidism in Patients with Prolonged Lithium Therapy," World Journal of Surgery, Vol. 27, No. 4, 2003, pp. 486-488. doi:10.1007/s00268-002-6824-4

[6] N. Perera, L. Gluch and B. A. Crawford, "Misleading Parathyroid Sestamibi Scan in Lithium Users," Internal Medicine Journal, Vol. 39, No. 8, 2009, pp. 556-557. doi:10.1111/j.1445-5994.2009.01972.x

[7] J. I. Lew and C. C. Solorzano, "Surgical Management of Primary Hyperparatyroidism: State of the Art," Surgical Clinics of North America, Vol. 89, No. 5, 2009, pp. 1205-1225. doi:10.1016/j.suc.2009.06.014

[8] H. Bendz, I. Sjödin, G. Toss and K. Berglund, "Hyperparathyroidism and Long-Term Lithium Therapy: A Cross-Sectional Study and the Effect of Lithium Withdrawal," Journal of Internal Medicine, Vol. 240, No. 6, 1996, pp. 357-365. doi:10.1046/j.1365-2796.1996.28864000.x

[9] L. E. Mallette, K. Khouri, H. Zengotita, B. W. Hollis and S. Malini, "Lithium Treatment Increases Intact and Midregion Parathyroid Hormone and Parathyroid Volume," The Journal of Clinical Endocrinology \& Metabolism, Vol. 68, No. 3, 1989, pp. 654-660. doi:10.1210/jcem-68-3-654

[10] P. E. Garfinkel, C. Ezrin and H. C. Stancer, "Hypothyroidism and Hyperparathyroidism Associated with Lithium," Lancet, Vol. 2, 1973, pp. 331-332. doi:10.1016/S0140-6736(73)90846-5

[11] C. Christiansen, P. C. Baastrup and I. Transbol, "Development of Primary Hyperparathyroidism during Lithium Therapy: Longitudinal Study," Neuropsychobiology, Vol. 6, No. 5, 1980, pp. 280-283. doi:10.1159/000117770

[12] A. Szalat, H. Mazeh and H. R. Freund, "Lithium-Associated Hyperparathyroidism: Report of Four Cases and Review of the Literature," European Journal of Endocrinology, Vol. 160, No. 2, 2009, pp. 317-323. doi:10.1530/EJE-08-0620

[13] C. R. McHenry, F. Racke, M. Meister, P. Warnaka, M. Sarasua, E. F. Nemeth, et al., "Lithium Effects on Dispersed Bovine Parathyroid Cell Grown in Tissue Culture," Surgery, Vol. 110, No. 6, 1991, pp. 1061-1066.

[14] J. Järhult, S. Ander, B. Asking, S. Jansson, A. Meehan, A. Kristoffersson, et al., "Long-Term Results of Surgery for Lithium-Associated Hyperparathyroidism," British Journal of Surgery, Vol. 97, No. 11, 2010, pp. 1680-1685.

[15] W. Khairallah, A. Fawaz, E. M., Brown and G. El-Hajj Fuleihan, "Hypercalcemia and Diabetes Insipidus in a Patient Previously Treated with Lithium," Nature Clinical 
Practice Nephrology, Vol. 3, No. 7, 2007, pp. 397-404. doi:10.1038/ncpneph0525

[16] E. Carchman, J. Ogilvie, J. Holst, J. Yim and S. Carty, "Appropriate surgical Treatment of Lithium-Associated Hyperparathyroidism," World Journal of Surgery, Vol. 32, No. 10, 2008, pp. 2195-2199. doi:10.1007/s00268-008-9616-7

[17] H. Abdullah, R. Bliss, A. I. Guinea and L. Delbridge, "Pathology and Outcome of Surgical Treatment for Lithium-Associated Hyperparathyroidism," British Journal of Surgery, Vol. 86, No. 1, 1999, pp. 91-93. doi:10.1046/j.1365-2168.1999.00977.x

[18] J. C. Hundley, D. T. Woodrum, B. D. Saunders, G. M. Doherty and P. G. Gauger, "Revisiting Lithium-Associated Hyperparathyroidism in the Era of Intraoperative Parathyroid Hormone Monitoring," Surgery, Vol. 138, No. 6, 2005, pp. 1027-1031. doi:10.1016/j.surg.2005.09.028

[19] J. Nordenström, M. Elvius, M. Bågedahl-Strindlund, B. Zhao and O. Törring, "Biochemical Hyperparathyroidsm and Bone Mineral Status in Patients Treated Long-Term with Lithium," Metabolism, Vol. 43, No. 12, 1994, 15631567. doi:10.1016/0026-0495(94)90017-5

[20] J. Ananth and S. E. Dubin, "Lithium and Symptomatic Hyperparathyroidism," Journal of the Royal Society of Medicine, Vol. 76, No. 12, 1983, pp. 1026-1029.

[21] M. E. Wolf, M. Moffat, J. Mosnaim and S. Dempsey, "Lithium Therapy, Hypercalcemia, and Hyperparathyroidism," American Journal of Therapeutics, Vol. 4, No. 9-10, 1997, pp. 323-325. doi:10.1097/00045391-199709000-00007

[22] G. de Celis, M. Fiter, X. Latorre, and C. Llebaria, "Oxyphilic Parathyroid Adenoma and Lithium Therapy," Lancet, Vol. 352, No. 9133, 1998, p. 1070. doi:10.1016/S0140-6736(05)60117-1

[23] J. A. Sloand, M. A and M. A. Shelly, "Normalization of Lithium-Induced Hypercalcemia and Hyperparathyroidism with Cinacalcet Hydrochloride," American Journal of Kidney Diseases, Vol. 48, No. 5, 2006, pp. 832-837. doi:10.1053/j.ajkd.2006.07.019

[24] H. M. Khandwala and S. Van Uum, "Reversible hypercalcemia and Hyperparathyroidism Associatead with Lithiuim Therapy: Case Report and Review of Literature," Endocrine Practice, Vol. 12, No. 1, 2006, pp. 5458.

[25] M. Rifai, J. K., Moles and D. P. Harrington, "LithiumInduced Hypercalcemia and Parathyroid Dysfunction," Psychosomatics, Vol. 42, No. 4, 2001, pp. 359-361. doi:10.1176/appi.psy.42.4.359

[26] H. S. Duggal and I. Singh, "Lithium-Induced Hypercalcemia and Hyperparathyroidism Presenting with Delir- ium," Prog Neuropsychopharmacol Biol Psychiatry, Vol. 32, No. 3, 2008, pp. 903-904. doi:10.1016/j.pnpbp.2007.12.014

[27] M. M. Rizwan and N. D. Perrier, "Long-Term Lithium Therapy Leading to Hyperparathyroidism: A Case Report," Perspect Psychiatr Care, Vol. 45. No. 1, 2009, pp. 62-65.

[28] S. Carney and P. Jackson, "Acute Lithium Administration Impairs the Action of Parathyroid Hormone on Rat Renal Calcium Magnesium and Phosphate Transport," Clinical and Experimental Pharmacology and Physiology, Vol. 25, No. 10, 1998, pp. 795-799. doi:10.1111/j.1440-1681.1998.tb02155.x

[29] D. Riccardi and G. Gamba, "The Many Roles of the Calcium-Sensing Receptor in Health and Disease," Archives of Medical Research, Vol. 30, No. 6, 1999, pp. 436-448. doi:10.1016/S0188-4409(99)00071-5

[30] C. Livingstone and H. Rampes, "Lithium: A Review of Its Metabolic Adverse Effects," Journal of Psychopharmacology, Vol. 20, No. 3, 2006, pp. 347-355. doi:10.1177/0269881105057515

[31] F. H. Shen and D. J. Sherrard, "Lithium-Induced Hyperparathyroidism: An Alteration of the 'Set Point'," Annals of Internal Medicine, Vol. 96, No. 1, 1982, pp. 63-65.

[32] E. M. Grandiean and J. M. Aubry, "Lithium: Updated Human Knowledge Using an Evidence-Based Approach: Part III: Clinical Safety," CNS Drugs, Vol. 23, No. 5, 2009, pp. 397-418. doi:10.2165/00023210-200923050-00004

[33] A. W. Saxe and G. Gibson, "Lithium Increases Tritiated Thymidine Uptake by Abnormal Human Parathyroid Tissue," Surgery, Vol. 110, No. 6, 1991, pp. 1067-1076

[34] P. S. Gregoor and G. M. de Jong, "Lithium Hypercalcemia, Hyperparathyroidism, and Cinacalcet," Kidney Int, Vol. 71, No. 5, 2007, p. 470. doi:10.1038/sj.ki.5002065

[35] L. A. Smith, V. Cornelius, A. Warnock, A. Bell and A. H. Young, "Effectiveness of Mood Stabilizers and Antipsychotics in the Maintenance Phase of Bipolar Disorder: A Systematic Review of Randomized Controlled Trials," Bipolar Disorders, Vol. 9, No. 4, 2007, pp. 394-412. doi:10.1111/j.1399-5618.2007.00490.x

[36] A. H. Young and J. M. Hammond, "Lithium in Mood Disorders: Increasing Evidence Base, declining Use?" The British Journal of Psychiatry, Vol. 191, 2007, pp. 474-476. doi:10.1192/bjp.bp.107.043133

[37] M. A. Kouvaraki, M. Greer, S. Sharma, D. Beery, R. Armand, J. E. Lee, et al., "Indications for Operative Intervention in Patients with Asymptomatic Primary Hyperparathyroidism: Practice Patterns of Endocrine Surgery," Surgery, Vol. 139, No. 4, 2006, pp. 527-534. doi:10.1016/j.surg.2005.09.006 